\title{
Outdooraktivitäten und damit zusammenhängende Einrichtungen im Winter
}

\author{
Coordinating Lead Authors (CLAs) \\ Robert Steiger \\ Lead Authors (LAs) \\ Robert Steiger, Ulrike Pröbstl-Haider, Franz Prettenthaler \\ Contributing Authors (CAs) \\ Andrea Damm, Martin Falk, Christoph Neger
}

\subsection{Allgemeine Entwicklung und Trends}

Wintertourismus in Österreich war im 20. Jahrhundert meist weniger bedeutend als der Sommertourismus (Abb. 1.2). Eine Angleichung der Nächtigungszahlen vollzog sich erst im Laufe der 1980er- und 1990er-Jahre. Der Anteil der Winternächtigungen an den Gesamtnächtigungen lag im Jahr 2018 bei $48 \%$ und hat sich seit dem Jahr 2000 nur geringfügig verändert. Jedoch sind die Tagesausgaben der Gäste im Winter höher $(185 €)$ als im Sommer (160€; Österreich Werbung 2018e, 2019; Aufschlüsselung siehe Tab. 7.1). Wichtig wäre in diesem Zusammenhang eine Betrachtung der Unterschiede in der Wertschöpfung des Winter- bzw. Sommertourismus. Daten und Publikationen hierzu sind allerdings nicht bekannt. In den westlichen Bundesländern Vorarlberg, Tirol und Salzburg werden seit Mitte bis Ende der 1980erJahre im Winter (November-April) mehr Übernachtungen generiert als im Sommer (Mai-Oktober). Österreichweit sind die Winternächtigungen in den 1980ern um durchschnittlich $2,5 \%$ pro Jahr gestiegen, in den 1990 ern um $0,7 \%$, in den 2000ern um 1,7 \% und im Zeitraum 2010-2018 um 1,3\% pro Jahr (Statistik Austria 2019). Der Wintersport spielt hierbei eine bedeutende Rolle: Rund $66 \%$ der Winternächtigungen entfallen auf Wintersportgemeinden (mit drei oder mehr Seilbahn-/Schleppliftanlagen; Fleischhacker 2018). Die Nächtigungsentwicklung in Wintersportgemeinden war jedoch im Zeitraum 1997/1998-2017/2018 weniger dynamisch (durchschnittlich $+1,28 \%$ pro Jahr) als in den übrigen Gemeinden $(+2,95 \%$ pro Jahr). Innerhalb der Wintersportregionen zeigen sich auch Verschiebungen der Marktanteile mit Verlusten im Süden zugunsten von Regionen im Westen Österreichs (Firgo und Fritz 2017).

Der österreichische Wintertourismus ist geprägt von einem hohen Stammgästeanteil von rund 77 \% (Österreich Werbung 2018a). Als Hauptaktivität wird von den Gästen das Skifahren (59\%) angegeben, gefolgt von Winterwandern (13\%) und
Snowboarden (9\%). Langlaufen wird nur von $3 \%$ der Gäste als Hauptaktivität betrieben (Österreich Werbung 2018a, b, c, d). Es besteht somit eine klare Dominanz des Schneesports. Ein Vergleich dieser Zahlen von 2018 mit 2012 zeigt einen Rückgang des Skisports von ehemals 65 auf $59 \%$ sowie einen Anstieg des Winterwanderns von 10 auf $13 \%$. Das Durchschnittsalter der Wintergäste ist in diesem Zeitraum von 42,6 Jahren auf 45,3 Jahre angestiegen (Österreich Werbung 2012, 2018a). Mögliche Gründe für diese Veränderungen sind der demografische Wandel in den Hauptherkunftsländern, sinkendes Interesse am Skifahren in den jüngeren Bevölkerungsschichten sowie eine breitere Angebotspalette und damit eine Veränderung der Gästestruktur.

Die Mehrheit reist mit dem Auto an (76\%), gefolgt vom Flugzeug (13\%), der Bahn (7 \%) und mit Reisebussen (3\%; Österreich Werbung 2018a). Der vergleichsweise geringe Anteil des Flugzeugs ist grundsätzlich positiv zu werten, da dieser Verkehrsträger die mit Abstand größten Treibhausgasemissionen verursacht. Jedoch hat sich dieser Anteil seit 2012 um 4 Prozentpunkte erhöht (Österreich Werbung 2012). Der Anteil der mit der Bahn anreisenden Wintergäste ist seit 2012 unverändert. Es wird zu beobachten sein, wie sich dieser Anteil künftig verändern wird. Andererseits deutet der immer noch sehr geringe Anteil der Nutzung öffentlicher Verkehrsmittel auf ein hohes Optimierungspotenzial hin. Eine verstärkte Zusammenarbeit mit Bahnen und Wintersportgemeinden, attraktive Preisangebote und öffentliche Investitionen in Eisenbahnen sind wichtig, um die Attraktivität des öffentlichen Verkehrs zu erhöhen. Dies könnte auch die Verkehrsbelastung vor allem zum Urlauberschichtwechsel in vielen Zielgebieten verringern.

Die österreichischen Skigebiete verzeichneten in der Saison 2017/2018 54,6 Mio. Ersteintritte (WKO 2019) und liegen damit weltweit nach den USA an zweiter Stelle (Vanat 2018). Die Entwicklung der letzten zehn Jahre mit einem durchschnittlichen jährlichen Wachstum von $0,4 \%$ 
deutet hierbei auf eine Stagnation auf hohem Niveau, mit gewissen jährlichen Schwankungen, hin (WKO 2001, 2007 und 2018). Ein solcher Prozess ist typisch für die späte Phase des Produkt- und Destinationslebenszyklus, der mit einer Verschärfung des Wettbewerbs einhergeht (Butler 1980).

Die anspruchsvoller werdende Wettbewerbssituation spiegelt sich auch in der finanziellen Situation der Skigebiete wider: Nur $45 \%$ der Skigebiete in Österreich wiesen in der Bilanz 2013 einen Gewinn aus (Falk und Steiger 2019). Die durchschnittliche Verschuldungsquote (Verhältnis zwischen Fremd- und Eigenkapital) von rund 250 untersuchten Skigebieten beträgt 73 und $23 \%$ der Unternehmen weisen eine negative Eigenkapitalposition auf (Falk und Steiger 2018). Vor allem die Größe und Höhenlage der Skigebiete sind wichtige Indikatoren für die Schuldenquote, mit bis zu 40 Prozentpunkten Unterschied zwischen kleinen und großen bzw. niedrig und hoch gelegenen Skigebieten. Im Zeitraum 1995-2011 sind $20 \%$ der Skigebiete vorübergehend in Konkurs gegangen, einige wenige sind gänzlich vom Markt verschwunden, welche aber nur $2 \%$ der Gesamtpistenfläche ausmachen (Falk 2013a).

Bei der Analyse von permanenten Schließungen zeigt sich, dass eine frühzeitige Einführung von Beschneiungsanlagen und die Größe der Skigebiete zu einem geringeren Ausfallrisiko führen. Die frühzeitige Einführung von Beschneiungsanlagen kann jedoch nicht das Risiko einer vorübergehenden Schließung oder die Wahrscheinlichkeit einer Insolvenz verringern. Hoch gelegene Skigebiete (mittlere Höhe von $1700 \mathrm{~m}$ ) weisen ein wesentlich geringeres Risiko auf. Insgesamt deuten die Ergebnisse darauf hin, dass Skigebiete ohne Beschneiungsanlagen und/oder mit einer geringen Größe eher vom Markt verdrängt werden (Falk 2013a). Eine mögliche Reaktion der Betreiber, die seit den 2000ern vermehrt verfolgt wird, sind Skigebietszusammenschlüsse. Diese führten im Mittel zu einer Erhöhung der Übernachtungen von $12 \%$. Der positive Nächtigungseffekt war jedoch bei Zusammenschlüssen vor 2004/2005 noch etwas höher (14\%) als im Zeitraum 2005/2006-2014/2015 (10 \%; Falk 2017). Ob neue Zusammenschlüsse den gleichen Effekt erzielen, ist fraglich. Zudem sind derartige Zuwächse auch unter dem Aspekt regionaler Verlagerungseffekte zu betrachten, welche auf eine räumliche Konzentration des Tourismus in Österreich hinweisen (Bätzing 2017). Hierbei zu berücksichtigen sind auch die ökologischen Folgen sowie der erwartbare zusätzliche Nutzen für den Gast (z. B. reine Verbindungsbahnen ohne Pisten).

\subsection{Relevante Entwicklungen in den wichtigsten Herkunftsländern}

Die wichtigsten Herkunftsländer des österreichischen Wintertourismus sind Deutschland (38\%) und Österreich (23\%). Die Marktanteile der nächstgereihten Quellmärkte Nieder- lande (8 \%), Schweiz und Großbritannien (je $3 \%$ ) sind schon deutlich geringer (Österreich Werbung 2018a). In Folge werden die Entwicklungen in den mit Abstand wichtigsten Quellmärkten Deutschland und Österreich betrachtet.

Der Anteil der österreichischen Bevölkerung, die regelmäßig Ski fährt, ist seit den 1980ern (13\%) auf $5 \%$ im Jahr 2017 gesunken (Zellmann und Mayrhofer 2018b). Der Anteil der zumindest gelegentlich skifahrenden Bevölkerung ist mit $27 \%$ jedoch deutlich höher (Zellmann und Mayrhofer 2010). Der Anteil der Nichtskifahrer hat seit den 1980erJahren (42\%) deutlich zugenommen (63\%; Zellmann und Mayrhofer 2018a).

Im deutschen Quellmarkt hat sich der Anteil der Befragten, die schon einmal Wintersport ausgeübt haben von $52 \%$ im Jahr 2012 auf 64 \% im Jahr 2018 erhöht (Roth et al. 2018). Die Hauptaktivität ist das Winterwandern (knapp 40 \%) gefolgt von Skifahren (38\%). Auch hier zeigen sich deutliche Veränderungen: Das Winterwandern hat um knapp 10 Prozentpunkte zugelegt, während das Skifahren um rund 7 Prozentpunkte abgenommen hat (Roth et al. 2012, 2018), d. h., das Winterwandern hat Skifahren als sportliche Hauptaktivität im Winter abgelöst. Ein direkter Vergleich der genannten Zahlen aus Österreich mit dem deutschen Quellmarkt ist nicht möglich, da unterschiedliche Aspekte erhoben wurden (,regelmäßig Ski fahren“ bzw. ,schon einmal Wintersport ausgeübt"). Beide Studien zeigen aber eine Veränderung der Hauptaktivitäten zulasten des Pistensports und zugunsten des Winterwanderns. Dies ist konsistent mit den ermittelten Veränderungen bei den Hauptaktivitäten von Winterurlaubern in Österreich. Skitouren sind in diesen Zahlen nicht enthalten. Genaue Zahlen sind hier nicht verfügbar.

\subsection{Einfluss des Klimawandels bzw. Einfluss auf den Klimawandel}

\subsubsection{Einfluss des Klimawandels}

Für schneebasierte touristische Angebote bewirkt der Klimawandel in niedrigen Lagen einen späteren Saisonstart und ein früheres Saisonende und führt somit insgesamt zu einer kürzeren Saisondauer (Steiger et al. 2019; vgl. auch Abschn. 2.2.2). Aufgrund der starken Saisonalität der Nachfrage innerhalb der Wintersaison, wird diese Entwicklung vermutlich erst dann relevant, wenn zum einen Perioden mit traditionell hoher Nachfrage (z. B. Weihnachten) betroffen sind (Steiger und Scott 2020). Zum anderen erhöht sich auch das Risiko von Schneemangel und damit verbunden das Risiko einer verminderten Qualität bis hin zu Ausfall des Angebots oder von Angebotsteilen in den Wintermonaten. Das Ausmaß dieser Änderungen ist höhen- sowie regionsabhängig (Steiger und Abegg 2013). Während bei Aktivitäten, die sich auf bewirtschaftete Flächen beschränken (z. B. Skifahren, Langlaufen, 
Tab. 6.1 Anteil schneesicherer Skigebiete in Österreich bei unterschiedlichen Indikatoren und Klimaszenarien. (Nachdruck aus Steiger und Scott 2020, mit Genehmigung von Elsevier)

\begin{tabular}{|c|c|c|c|c|c|c|c|}
\hline \multirow[t]{2}{*}{ Indikatoren } & \multirow{2}{*}{$\begin{array}{l}1981-2010 \\
{[\%]}\end{array}$} & \multicolumn{3}{|l|}{ RCP 4.5} & \multicolumn{3}{|l|}{ RCP 8.5} \\
\hline & & $2030 \mathrm{er}[\%]$ & $2050 \mathrm{er}[\%]$ & $2080 \mathrm{er}[\%]$ & $2030 \mathrm{er}[\%]$ & $2050 \mathrm{er}[\%]$ & $2080 \mathrm{er}[\%]$ \\
\hline $\begin{array}{l}\text { 100-Tage-Indikator mit } \\
\text { heutiger Beschneiungs- } \\
\text { kapazität }\end{array}$ & 90 & 80 & 72 & 54 & 78 & 52 & 11 \\
\hline $\begin{array}{l}\text { 100-Tage-Indikator mit } \\
\text { verbesserter Beschnei- } \\
\text { ungskapazität }(10 \mathrm{~cm} / \mathrm{Tag})\end{array}$ & 99 & 93 & 92 & 83 & 93 & 80 & 31 \\
\hline $\begin{array}{l}\text { Schneesicher in den Weih- } \\
\text { nachtsferien mit heutiger } \\
\text { Beschneiungskapazität }\end{array}$ & 84 & 65 & 52 & 37 & 63 & 33 & 5 \\
\hline $\begin{array}{l}\text { Schneesicher in den } \\
\text { Weihnachtsferien mit ver- } \\
\text { besserter Beschneiungs- } \\
\text { kapazität }(10 \mathrm{~cm} / \mathrm{Tag})\end{array}$ & 98 & 90 & 80 & 67 & 92 & 66 & 15 \\
\hline
\end{tabular}

Rodeln), bei entsprechender Wertschöpfung, mit technischer Beschneiung die Variabilität der natürlichen Schneedecke ausgeglichen werden kann, sind Aktivitäten, wie z. B. Skitourengehen abseits der Pisten, Schneeschuhwandern, abhängig von der natürlichen Schneelage und somit potenziell stärker vom Klimawandel betroffen.

Auswirkungen des Klimawandels auf den Skitourismus sind aufgrund der hohen wirtschaftlichen Bedeutung weltweit vergleichsweise gut untersucht (Steiger et al. 2019). Generell zeigt sich, dass die Auswirkungen regional sehr unterschiedlich sein können, mit Skigebieten, die vergleichsweise wenig vom Klimawandel betroffen sein werden, bis hin zu Skigebieten, in denen starke Veränderungen zu erwarten sind.

Für die Bewertung der Klimawandelfolgen wird oftmals die Schneesicherheit von Skigebieten herangezogen. Diese ist gegeben, wenn ein Skibetrieb von mindestens 100 Tagen in zumindest sieben von zehn Jahren gewährleistet werden kann (Abegg 1996). Nach dieser Regel wurden in einer OECD-Studie (Abegg et al. 2007) 228 österreichische Skigebiete untersucht. Hierbei wurde festgestellt, dass der Osten Österreichs früher mit negativen Folgen für die Skigebiete zu rechnen hat als der Westen. Allerdings ist davon auszugehen, dass der potenzielle regionalwirtschaftliche Schaden im Westen aufgrund der größeren Abhängigkeit vom Skitourismus höher ausfällt als im Osten (Breiling et al. 1997). Durch die starke Höhenabhängigkeit der Ergebnisse ist die Verwundbarkeit Österreichs hierbei größer als die der Schweiz, Frankreichs und Italiens und geringer als die Deutschlands. Allerdings wurden in der OECD-Studie die erheblichen klimatischen Unterschiede in den Alpen nur recht grob (Steiger 2010), die Anzahl und Qualität der Beschneiungsanlagen gar nicht berücksichtigt (Mayer et al.
2007; Steiger und Mayer 2008). Während Ersteres zu einer vermeintlichen Vergleichbarkeit von Höhenlagen hinsichtlich der Schneesicherheit geführt hat (Steiger 2010), bewirkt Letzteres eine Überschätzung der möglichen Auswirkungen (Steiger und Abegg 2013).

Auch bei Berücksichtigung der gegenwärtigen Beschneiungskapazität in den Skigebieten zeigt sich im 21. Jahrhundert ein deutlicher Rückgang der schneesicheren Skigebiete im Vergleich zu dem Zeitraum 1981-2010 (Tab. 6.1). So wären Mitte des 21. Jahrhunderts im RCP-8.5-Szenario mit hohen Emissionen nur noch $52 \%$ der Skigebiete schneesicher. Eine Erhöhung der Schneileistung in allen Skigebieten auf die heutige Referenzgröße (Grundbeschneiung innerhalb 72 Stunden) kann die Schneesicherheit deutlich verbessern, mit zumindest noch $80 \%$ schneesicheren Skigebieten. Bei Betrachtung der touristisch sehr wichtigen Weihnachtsferien zeigt sich jedoch ein stärkerer Effekt des Klimawandels auf die Schneesicherheit (Tab. 6.1). Es wird also zunehmend schwieriger werden, den Skibetrieb in dieser frühen Phase des Winters sicherzustellen. Die dargestellten Auswirkungen (Steiger und Scott 2020) sind im Vergleich zu früheren Modellrechnungen (Steiger 2010; Steiger und Abegg 2013, 2015; Steiger und Stötter 2013) weniger gravierend. Gründe hierfür sind die Verwendung der aktuellsten ÖKS15-Klimaszenarien (Chimani et al. 2016), die Berücksichtigung der Exposition und eine angenommene höhere Grenztemperatur der Beschneiung $\left(-2{ }^{\circ} \mathrm{C}\right.$ in Steiger und Scott 2020 statt $-5^{\circ} \mathrm{C}$ der vorangegangenen Studien). Die regionalen Muster sind jedoch unverändert, d. h., frühere und stärkere Auswirkungen sind am Alpenrand (Vorarlberg, Tiroler Unterland, nördliches Salzburg, Ober- und Niederösterreich) zu erwarten (Abb. 6.1). Diese Ergebnisse zeigen zweierlei: Zum einen wird es ver- 

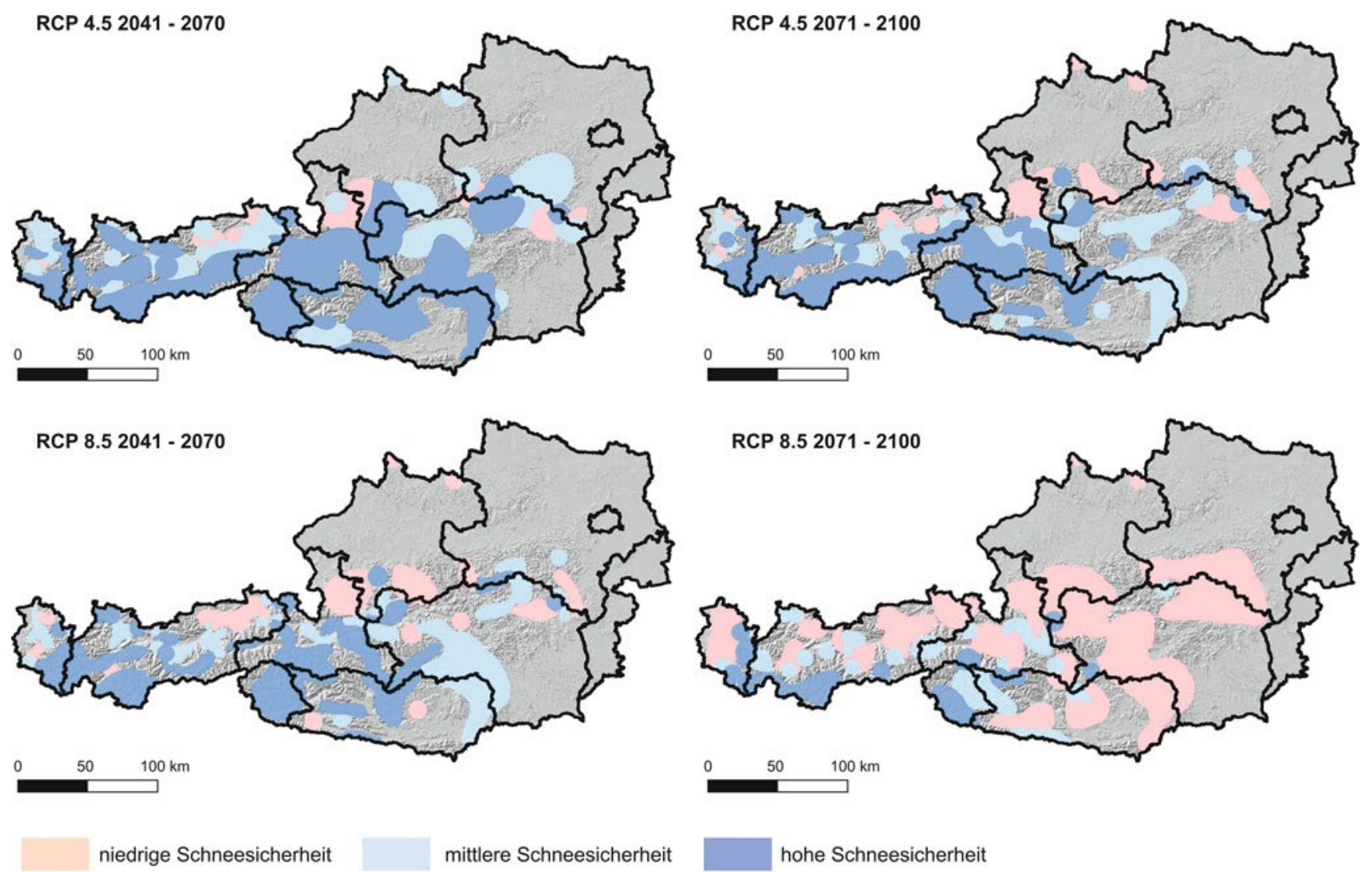

Abb. 6.1 Schneesicherheit von Skigebieten in Österreich mit verbesserter Beschneiungskapazität. (Datenquelle: Steiger und Scott 2020; Grafik: Moritz Waas)

mutlich auch Ende des 21. Jahrhunderts bei entsprechender Ausstattung mit Beschneiungsanlagen noch schneesichere Skigebiete geben. Dies ist jedoch mit einem deutlich höheren Beschneiungsaufwand, d. h. einem höheren Ressourcenbedarf und damit höheren Preisen verbunden. Zum anderen wird der Klimawandel den Druck auf weniger rentable Skigebiete tendenziell erhöhen, was in weiterer Folge zu einer stärkeren Konzentration und Marktbereinigung sowie einer künftig geringeren Anzahl an Skigebieten führen könnte. Große, hoch gelegene Skigebiete und solche mit guter Beschneiung sind bei dieser Entwicklung begünstigt. Allerdings zeigen Entwicklungen in den USA und in der Schweiz auch alternative Modelle (Pröbstl-Haider und Flaig 2019), wo zur Erhaltung der Einstiegs- und Übungsmöglichkeiten große, konkurrenzstarke Gebiete die Kleinen stützen, um langfristig die Nachfrage zu erhalten.

Für die Bewertung der Klimawandelfolgen für Skigebiete ist jedoch nicht nur die Angebotsseite relevant (also, ob schneesichere Verhältnisse hergestellt werden können), sondern auch die Nachfrageseite. Gäste haben grundsätzlich eine hohe Anpassungsfähigkeit, sowohl räumlich (Wahl eines anderen Skigebiets), zeitlich (Skifahren nur in Saisonzeiten mit ausreichend Schnee) und hinsichtlich der Aktivitäten (andere Aktivität statt Skifahren). Änderungen des Gästeverhaltens werden vermutlich eher rasch bei Erreichen von gewissen Grenzwerten (z. B. akzeptable Schneebedingungen) auftreten und nicht als kontinuierliche, langsame Veränderung (Gössling und Hall 2006).

Die Reaktion von Wintergästen auf schneearme Situationen wurde in mehreren Studien indirekt, also z. B. durch Analyse von Übernachtungsstatistiken, als auch direkt durch Gästebefragungen und Experimente untersucht. Für Winternächtigungen in Wintersportdestinationen Österreichs wurde ein Zusammenhang mit der Schneehöhe festgestellt, auch wenn dieser vergleichsweise schwach ausgeprägt ist und nur für niedrig gelegene Skigebiete gilt (maximale Höhe unter $2000 \mathrm{~m}$ ). Zudem ist dieser Effekt im betrachteten Zeitraum bis 2006/2007 schwächer geworden (Falk 2010; Töglhofer et al. 2011). Eine Erklärung für die abnehmende Abhängigkeit der Nächtigungen von der Naturschneehöhe ist die zugenommene Verbreitung von Beschneiungsanlagen (Falk und Lin 2018). Die Empfindlichkeit von Wintergästen gegenüber Klimaschwankungen ist bei inländischen Gästen stärker als bei ausländischen Gästen ausgeprägt, wenn auch das absolute Ausmaß der Empfindlichkeit recht gering ist (Falk 2013b). Bei Auswertung des Reiseverhaltens der Österreicher in den Dezembermonaten zwischen 2012 bis 2016 wurde festgestellt, dass auch ein sehr warmer Dezember keinen negati- 
ven Einfluss auf die Wahrscheinlichkeit eines Urlaubs in den Bergen hatte (Falk und Hagsten 2018).

Im sehr warmen und schneearmen Winter 2006/2007 (rund $3{ }^{\circ} \mathrm{C}$ über dem 30-jährigen Mittelwert) hatten die österreichischen Seilbahnen einen Umsatzrückgang von rund $7 \% \mathrm{zu}$ verzeichnen (WKO 2007). Kleine Skigebiete (Förderkapazität $<5000 \mathrm{P} / \mathrm{h}$ ) waren jedoch deutlich stärker betroffen mit durchschnittlichen Einbußen von $39 \%$ ebenso wie niedrig gelegene Skigebiete mit einer mittleren Höhe unter 1500 m mit Einbußen von durchschnittlich $22 \%$ (Steiger 2011).

Das Sommerskifahren als Spezialfall des alpinen Skisports ist seit ca. Mitte der 1980er-Jahre rückläufig (Abegg et al. 1994; Diolaiuti et al. 2006). Derzeit bietet in Österreich nur noch ein Gletscherskigebiet (Hintertuxer Gletscher) Skibetrieb den ganzen Sommer hinweg an. Die Gründe für den Rückgang des Sommerskifahrens sind zum einen angebotsseitig der Gletscherrückgang und die damit verbundene Verkleinerung des Angebots und ein zu starker Rückzug der sommerlichen Schneefläche auf den Gletschern (Abegg et al. 1994). Durch steigende Kosten als Folge leistungsfähigerer Bahnen werden für einen rentablen Betrieb mehr Gäste benötigt. Aber auch nachfrageseitig haben sich Änderungen ergeben: Andere Sommersportarten machten dem Sommerskifahren zunehmend Konkurrenz, zudem bestehen Vermutungen, dass das steigende Umweltbewusstsein in den 1980er-Jahren zum Nachfragerückgang beigetragen hat (Mayer et al. 2018).

Der Klimawandel wirkt sich durch auftauenden Permafrost (Abschn. 2.2.2) auf die Sicherungskosten der hochalpinen Infrastruktur aus. Beim Skibetrieb ergeben sich zunehmend Herausforderungen bei der Pistenführung über den Gletscher, da durch den Gletscherrückgang Felsriegel ausapern, die bestehende Pisten verschmälern und/oder unterbrechen können. Den Herbstbetrieb auf den Gletschern sicherzustellen ist somit ebenfalls mit einem höheren Aufwand verbunden.

Die Gletscherskigebiete stellen jedoch durch die hohe Schneesicherheit einen wichtigen Teil des Angebots in der Wintersaison dar. Für sechs von acht österreichischen Destinationen mit Gletscherskigebieten wurde festgestellt, dass die Übernachtungen in schneereichen Wintern unter-, in schneearmen Wintern dagegen überdurchschnittlich sind (Töglhofer et al. 2011; Mayer et al. 2018). Dies bedeutet, dass sich die Nachfrage in schneearmen Wintern etwas in Richtung Gletscher- und andere hoch gelegene Skigebiete verlagert. Destinationen mit Zugang zu einem Gletscherskigebiet innerhalb 20 Minuten Fahrdistanz werden von Gästen positiv bewertet (Pröbstl-Haider et al. 2015).

Gästebefragungen in Österreich ergaben, dass rund ein Viertel der Befragten bei unsicheren Schneeverhältnissen auf einen Skiurlaub verzichten würde (Unbehaun et al. 2008; Fleischhacker et al. 2009). Zugleich ist die Bereitschaft zur Aufgabe der Loyalität zu einer Destination zugunsten einer Destination mit besseren Schneeverhältnissen sehr hoch (Un- behaun et al. 2008). Ebenso gaben die Befragten an, dass sie in schneearmen Wintern weniger Ski fahren würden (Unbehaun et al. 2008; Luthe 2009). Rund ein Drittel der Skifahrer würde keinen Skiausflug oder Skiurlaub machen, wenn nur die Hälfte der Pisten geöffnet ist (Steiger und Posch 2017). Für $21 \%$ der Tagesgäste und $19 \%$ der Urlaubsgäste ist ein Szenario mit kaum Naturschnee, aber beschneiten Pisten nicht akzeptabel. Für Tagesgäste sind die aktuellen Naturschneebedingungen entscheidend für die Destinationswahl, knapp gefolgt von der Anreisezeit. Für Urlaubsgäste dagegen sind vergangene Erfahrungen mit der Schneelage sowie die Schneesicherheit am wichtigsten (Steiger et al. 2020). Bei der Bedeutung der Schneesicherheit wurden jedoch Unterschiede zwischen Nationen festgestellt, wobei Österreicher mehr Wert auf Schneesicherheit legten als deutsche oder italienische Wintersportler (Pröbstl-Haider und Mostegl 2019). Skifahrer mit einem höheren Fahrkönnen wechseln eher die Destination, sind also weniger loyal als weniger gute Skifahrer, Anfänger dagegen entscheiden sich eher dazu, gar nicht Ski zu fahren (Pröbstl-Haider et al. 2015; Rutty et al. 2015a).

Die Folgen einer kürzeren Skisaison in manchen Jahren verbunden mit den daraus resultierenden Gästereaktionen wurden mit Nachfragemodellen untersucht. So könnte eine Erwärmung um $2{ }^{\circ} \mathrm{C}$ in einem schneearmen Winter, im Vergleich zu einem durchschnittlichen Winter in der Referenzperiode 1971-2000, zu Einbußen von 3,4-4,1 Mio. Nächtigungen in Österreich führen (Damm et al. 2017). In einer weiteren Studie wurden die potenziellen Verluste für die Tourismuswirtschaft bei moderatem Klimawandel (mittleres Szenario) für den Zeitraum 2016-2045 auf rund 90 Mio. $€$ pro Jahr geschätzt und für 2036-2065 auf rund 300 Mio. $€$ pro Jahr (Köberl et al. 2015). In beiden Fällen war die Analyse allerdings auf Nachfrageveränderungen bei den Nächtigungen durch Naturschneemangel beschränkt. Der Effekt der technischen Beschneiung auf die Erstzutritte eines Skigebietes wurden in Damm et al. (2014) untersucht. Bis 2050 ist ein klimawandelbedingter Rückgang der Erstzutritte von 6 bis $28 \%$ zu erwarten, während bei reiner Naturschneebetrachtung ein Rückgang von 22 bis $64 \%$ berechnet wurde. Insgesamt zeigt sich auch die deutlich höhere Klimasensitivität bei (Tages-)Skigästen im Vergleich zu Nächtigungsgästen. Grundsätzlich unberücksichtigt sind in diesen Studien schneeunabhängige Zusatzangebote, welche die dargestellten Verluste unter Umständen reduzieren könnten.

Durch die große Anpassungsfähigkeit des Gastes ist es wahrscheinlich, dass sich zuerst Veränderungen der Nachfrageverteilung innerhalb eines Marktes in Richtung Gunstregionen bemerkbar machen (Rutty et al. 2015b). Dies könnte $\mathrm{zu}$ einer weiteren Konzentration des Tourismus führen mit entsprechenden Herausforderungen in begünstigten Destinationen hinsichtlich Kapazität, ökologischer und sozialer Tragfähigkeit, Verkehr etc. Eine Simulation von Nachfrageverschiebungen, basierend auf Schneemodellierungen (Steiger 
und Scott 2020) und Gästebefragungen (Steiger und Posch 2017), zeigt eine nur geringe Änderung der Nachfrage auf nationaler Ebene bis Mitte des Jahrhunderts (Steiger et al. 2018). Unter Annahme einer Erhöhung der Beschneiungskapazität in allen Skigebieten auf den heutigen Referenzwert (72 Stunden Grundbeschneiung) würde sich die Gästezahl in den Skigebieten bis 2050 nur um 2,2-6,7 \% verringern (RCP 4.5 bzw. RCP 8.5), trotz angenommener Schließung von nichtschneesicheren Skigebieten. Auf regionaler Ebene hingegen zeigen sich deutliche Verschiebungen der Nachfrage, welche von Verlusten über $50 \%$ (z. B. Regionen am Alpenrand) bis hin zu Zuwächsen von 25-50 \% (z. B. im westlichen Tirol, Osttirol, Teile Kärntens) reichen. Gegen Ende des Jahrhunderts beträgt der österreichweite Rückgang im mittleren Szenario (RCP 4.5) 5,7 \%, im „business-as-usual“-Szenario (RCP 8.5) dagegen $63,4 \%$ (Steiger et al. 2018).

Grundsätzlich ist der zu erwartende wirtschaftliche Nachteil durch Folgen des Klimawandels somit deutlich höher und geht zudem über die Tourismusbranche hinaus. Unter Annahme eines zehnprozentigen Nächtigungsrückgangs bei Berücksichtigung weiterer Effekte in anderen, vom Tourismus abhängigen Wirtschaftsbranchen (mithilfe eines multiregionalen Input-Output-Modells) wird der Effekt auf die Bruttowertschöpfung mit Verlusten von $32 \mathrm{Mrd}$ € geschätzt (Prettenthaler et al. 2009). Naturgemäß sind die Tourismusbundesländer und speziell das Beherbergungs- und Gaststättenwesen am stärksten direkt betroffen. Bemerkenswert ist jedoch, dass auch industriegeprägte Bundesländer durch Vorleistungsverflechtungen von indirekten Effekten sehr stark betroffen sind. Hier sind vor allem Oberösterreich und Niederösterreich zu nennen, in welchen die Lebensmittelindustrie, der Handel und das Bauwesen wichtige Wirtschaftssektoren sind, die von der Tourismusbranche nachgefragt werden (Prettenthaler und Formayer 2011). Diese indirekten Effekte sind vor allem in der mittleren Frist stärker spürbar.

$\mathrm{Zu}$ den Auswirkungen des Klimawandels auf weitere Winteraktivitäten neben dem Pistensport existieren noch vergleichsweise wenige Studien. Für das Langlaufen wurde festgestellt, dass das Thema Landschaftsästhetik, worunter auch die verschneite Winterlandschaft einzuordnen ist, für die Mehrheit der Langläufer in Österreich einen hohen Stellenwert hat (Landauer und Pröbstl 2008). Schneemangel wirkt sich somit bei Langläufern nicht nur direkt auf die Saisonlänge und die Möglichkeit, den Sport auszuüben, aus, sondern auch indirekt, indem eine Landschaft mit wenig oder keinem Naturschnee als weniger attraktiv wahrgenommen wird. Im Falle schlechter Schneebedingungen ist die Wahl einer schneesicheren Langlaufdestination die bevorzugte Anpassungsmaßnahme, gefolgt von einer kurzfristigeren Urlaubsbuchung, wenn genügend Schnee liegt, und dem Wechsel von Langlaufurlaub zu Tagesausflügen bei guter Schneelage (Landauer und Pröbstl 2008). Rund 20 \% würden den Sport bei ungenügenden Verhältnissen eher nicht ausüben, wohingegen das gesundheitsaffine Segment (55 \%) am robustesten gegenüber Schneemangel ist und bereit wäre, auf andere Sportarten in der Destination auszuweichen (Landauer et al. 2012; Pröbstl-Haider und Haider 2013). Bauliche Maßnahmen, wie z. B. die in Finnland populären Skitunnels, werden von den befragten österreichischen Langläufern deutlich abgelehnt (Landauer et al. 2013). Grundsätzlich ist jedoch auch zu berücksichtigen, dass Langlaufloipen zunehmend beschneit werden oder mit über den Sommer gelagertem Schnee im Spätherbst geöffnet werden. Diese punktuell feststellbare Zunahme (z. B. Davos, Seefeld) deutet auf vorhandene Kundensegmente hin, die ein derartiges Angebot nachfragen.

Eissportarten sind ebenfalls potenziell vom Klimawandel betroffen. Bei steigenden Temperaturen ist zu erwarten, dass sich Nutzungszeiten von z. B. Outdooreisflächen verkürzen. Mögliche Folgen daraus sind technische Anpassungen, z. B. leistungsstärkere Kühlanlagen oder künstliche Beschattung (Überdachung), oder die Schließung von Outdooranlagen und gegebenenfalls Errichtung von Indooranlagen. Untersuchungen hierzu sind jedoch nicht bekannt.

\subsubsection{Einfluss auf den Klimawandel}

Ebenso wie alle anderen Tourismussegmente trägt auch der winterliche Outdoortourismus selbst zum Klimawandel bei. Dabei fällt der Hauptanteil der Treibhausgasemissionen, die von Wintertouristinnen und -touristen verursacht werden, auf Transport, Unterkunft und Verpflegung. In einer Studie von Friesenbichler (2003) zum Wintertourismus in Österreich (auf Basis von Daten aus dem Jahr 2001) machten zum Beispiel die Kategorie Beherbergung und Gastronomie 58,3\% und die Transportdienstleistungen 37,9\% aus, während die spezifische Wintersportinfrastruktur nur für 3,8\% verantwortlich war. In einer ähnlichen Studie in einem französischen Wintersportgebiet kamen Duprez und Burget (2007) auf 74,0 \% für An- und Abreise und Transport am Urlaubsort sowie 18,7 \% für Beherbergung und Gastronomie. Der Pistenservice machte dagegen nur $1,9 \%$ aus $(5,4 \%$ beliefen sich auf sonstige Aktivitäten, wie z. B. die Beheizung und Elektrizität von Geschäften oder die mit dem Tourismus verbundenen Transporte der örtlichen Bevölkerung). In einer jüngeren Studie des Umweltbundesamts (2018) fielen 50 \% der Emissionen für einen Skiurlaub in Österreich auf die Anund Abreise, $32 \%$ auf die Beherbergung und $18 \%$ auf die Aktivitäten. Diese Studien können nur generelle Tendenzen wiedergeben und sind auch nicht ohne Weiteres miteinander vergleichbar, da die Ergebnisse z. T. nur auf einem (Duprez und Burget 2007) oder wenigen Skigebieten (Friesenbichler 2003) beruhen, die dahinterliegenden Daten zum Teil schon recht veraltet sind (Friesenbichler 2003), oder vereinfachende Annahmen getroffen werden mussten (Umweltbundesamt 2018). 
Der global beobachtete Trend hin zu kürzeren Aufenthalten (Gössling et al. 2018) betrifft auch den Wintertourismus in Österreich und hat entsprechende Auswirkungen auf den Beitrag des Tourismus zum Klimawandel. Lag die durchschnittliche Aufenthaltsdauer im Winter in den 1980er-Jahren noch bei rund 6 Tagen, so wurde im Winter 2017/2018 ein neuer Tiefstwert mit 3,62 Tagen erreicht (Statistik Austria 2019). Die Aufenthaltsdauer im Zeitraum 2010-2018 lag um 34 \% niedriger als im Zeitraum 1980-1989. Um die Anzahl der Gäste und die damit verbundenen Einnahmen aus dem Tourismus zumindest halten zu können, ist folglich eine Erhöhung des Gästevolumens um den gleichen Betrag nötig. Da das Anreiseverhalten in diesem Zeitraum relativ stabil blieb, ergibt sich aus dieser nötigen Steigerung automatisch auch eine deutliche Verkehrszunahme. Die Ankünfte sind seit den 1980ern um rund $122 \%$ gestiegen (Statistik Austria 2019), haben sich also mehr als verdoppelt, mit entsprechenden Auswirkungen auf den Verkehr. Ein direkter Rückschluss auf durch touristischen Verkehr ausgelöste $\mathrm{CO}_{2}$-Emissionen ist nicht ohne Weiteres möglich, da sich der Durchschnittsverbrauch der Kraftfahrzeuge in diesem Zeitraum verringert hat. Zwischen 1995 und 2017 betrug in Deutschland dieser Rückgang 1,4 1/100 km oder 15,9 \% (BMVI 2018).

Es ist anzunehmen, dass im Wintertourismus die Emissionen durch Beheizung von Hotels und anderen Beherbergungsarten höher sind als bei anderen Tourismussegmenten. Genauere Vergleichsstudien dazu fehlen jedoch bislang. Angaben für Mobilität, Unterkunft sowie Gastronomie sind in den Kap. 3, 4 und 11 zu finden.

Die Beschneiung, und insbesondere der damit verbundene Energieverbrauch, wird in der Presse und Öffentlichkeit immer wieder im Zusammenhang mit dem Klimawandel thematisiert. Die österreichischen Seilbahnen geben den Strombedarf der Beschneiung mit $15.000 \mathrm{kWh}$ pro Jahr und Hektar an (WKO 2019). Bei rund 23.700 ha beschneiter Pistenfläche (WKO 2019) ergibt das 355,5 GWh. Andere Datenquellen gehen von deutlich mehr Strombedarf für die Beschneiung aus: Der deutsche Skiverband rechnet mit $20.000 \mathrm{kWh}$ pro Hektar für eine Grundbeschneiung von $30 \mathrm{~cm}$ (DSV 2019). Da die Grundbeschneiung in der Regel aber durch die Nachbeschneiung ergänzt wird (Steiger und Mayer 2008), dürften die Praxiswerte nochmals höher sein. Unter der Annahme, dass die Nachbeschneiung zusätzlich $30 \mathrm{~cm}$ beträgt (Pröbstl 2006), ergibt sich hieraus also ein Strombedarf von rund 355-950 GWh pro Jahr. Dies entspricht dem Stromverbrauch in privaten Haushalten von 215.000-570.000 Einwohnern (E-Control 2018). Diese Werte sind allerdings in Relation $\mathrm{zu}$ anderen Urlaubsformen zu sehen. So kam das UBA zum Schluss, dass bei einem Sommerurlaub in Österreich in etwa gleich viel Treibhausgasemissionen wie beim Winterurlaub anfallen, der Sommerurlaub in Italien hingegen ist in der Bilanz schon schlechter (Umweltbundesamt 2018). Allerdings ist hier zu beachten, dass die zugrunde liegenden Annahmen der Urlaubsaktivitäten und der durchschnittlichen Reisedistanz einen maßgeblichen Einfluss auf die Ergebnisse haben. Außerdem ist fraglich, ob der Strommix und somit die $\mathrm{CO}_{2}-$ Emissionen aus der Stromerzeugung im Winter gleich sind wie im Sommer. Daher können die dargestellten Ergebnisse nur eine grobe Abschätzung geben. Eindeutig ist jedoch, dass die Treibhausgasemissionen einer Flugreise die eines Winterurlaubs in Österreich um ein Vielfaches übersteigen.

Bezüglich der spezifischen Wintersportinfrastruktur lohnt es sich, nochmals einen Blick auf die Studie von Friesenbichler (2003) zu werfen, wo dieser Bereich genauer untersucht wird. Dabei werden folgende relevante Elemente identifiziert: Aufstiegshilfen, Beschneiungsanlagen, Pistengeräte und Flutlicht. Die Emissionen werden damit hauptsächlich im Bereich Alpinski, Snowboard und in geringerer Intensität im Bereich Langlauf erzeugt, während die technisch weniger aufwendigen Aktivitäten Winterwandern und Tourengehen kaum dazu beitragen. Mangels Daten zum Gesamtenergieverbrauch der österreichischen Skigebiete zog Friesenbichler zur Berechnung die Daten von ausgewählten Fallstudien heran. Vollständige Daten konnten dabei für die Planai-Hochwurzen-Bahnen in der Steiermark (Wintersaison 2000/2001) und für die Gletscherbahnen Kaprun (Geschäftsjahr 2000/2001) verwendet werden. Die Planai-Hochwurzen-Bahnen hatten in diesem Zeitraum 834.185 Ersteintritte zu verzeichnen. Das Skigebiet verfügte über eine Flutlichtanlage und Anlagen zur Beschneiung von 200 ha Pistenfläche. Für die Saison wurde unter Berücksichtigung des österreichischen Strommixes berechnet, dass $2926 \mathrm{t} \mathrm{CO}_{2}$ generiert wurden, davon 35,5\% durch die Beschneiungsanlagen, 35,2 \% durch Aufstiegshilfen, 25,9\% durch Pistengeräte, nur 0,1\% durch die Flutlichtanlage sowie 3,3 \% durch sonstigen Energieverbrauch, zum Beispiel zur Beheizung und Beleuchtung der Berg- und Talstationen. Die Gletscherbahnen Kaprun hatten 842.073 Ersteintritte, davon 736.233 in der Wintersaison. Die Beschneiungsfläche war in diesem Fall deutlich geringer, mit nur 18 ha. Flutlichtanlage gab es keine. Damit konnte gesamt die Erzeugung von 3166 t CO$_{2}$ geschätzt werden, 47,7 \% für Aufstiegshilfen, 29,8\% für Pistengeräte, 1,2 \% für die Beschneiung und $21,1 \%$ Sonstiges.

Damit beliefen sich die Emissionen pro Ersteintritt für die Planai-Hochwurzen-Bahnen auf 3,5 $\mathrm{kg} \mathrm{CO}_{2}$ und für die Gletscherbahnen Kaprun auf 3,8 kg. Für die Schweiz wurden 4-7 $\mathrm{kg} \mathrm{CO}_{2}$ pro Ersteintritt bei vier untersuchten Skigebieten festgestellt (Zegg et al. 2010). Für Österreich insgesamt berechnete Friesenbichler die Gesamtemissionen auf Basis einer Angabe des Fachverbandes für Seilbahnen zu den jährlichen Energieausgaben. Darauf basierend ging er von einem durchschnittlichen Wert pro Ersteintritt von nur 2,6 $\mathrm{kg} \mathrm{CO}_{2}$ aus, wodurch eine Emissionsmenge für die Outdoorinfrastruktur von 182.000 t $\mathrm{CO}_{2}$ geschätzt werden konnte und damit, wie bereits erwähnt, nur 3,8 \% der Gesamtemissionen im alpinen Wintertourismus ausmachte. 
Von 2000/2001 bis zur Wintersaison 2017/2018 hat die Pistenfläche in Österreich um $14 \%$ zugenommen und die Zahl der Beförderungen mit Aufstiegshilfen ist um $13 \%$ gestiegen (eigene Berechnungen auf Basis von WKO 2001, 2018). Die beschneite Pistenfläche wurde im selben Zeitraum von 29 auf $70 \%$ im Jahr 2018 erweitert (WKO 2001, 2018). Daher ist einerseits von steigenden Emissionen auszugehen, allerdings muss hierbei auch berücksichtigt werden, dass Beschneiungsanlagen sowie Bahnen auch energieeffizienter geworden sind. Eine Hochrechnung der Emissionen auf der oben dargestellten Basis ist daher ohne detailliertere Informationen nicht möglich. Zudem stellen die Berechnungen von Friesenbichler aufgrund der unzureichenden Datenverfügbarkeit nur einen groben Richtwert dar. $\mathrm{Zu}$ einer genaueren Berechnung wären vollständige Angaben zur Nutzung der Skigebiete in Österreich vonnöten. Dazu wäre es wünschenswert, nicht nur eine, sondern mehrere Wintersaisonen zu untersuchen, um auch meteorologisch bedingte Schwankungen in der Kunstschneeerzeugung zu berücksichtigen.

Eine Studie des Joanneum Research (Schwaiger et al. 2017) ist der Frage nachgegangen, inwieweit sich die höhere Reflexion von eingehender Strahlung über beschneiten Pistenflächen (Albedoeffekt) im Vergleich zur geringeren Rückstrahlung schneefreier Flächen auf die Klimabilanz der Beschneiung auswirkt. Ein Startclim-Projekt (Weihs und Laimighofer 2019) kam in Folge zum Schluss, dass die Kühlwirkung in Schwaiger et al. (2017) aufgrund einer zu stark vereinfachten Strahlungsmodellierung um das Sechsfache überschätzt wurde (Abschn. 2.3). Zudem müssten für die Bewertung der Klimawirksamkeit nicht nur $\mathrm{CO}_{2}$-Emissionen für die Beschneiung während des Betriebs berücksichtigt werden, sondern auch $\mathrm{CO}_{2}$-Emissionen, die im Zusammenhang mit der zusätzlichen Pistenpräparation, der Produktion, dem Transport und Aufbau der Beschneiungsanlagen stehen. Derartige Berechnungen existieren derzeit noch nicht.

Neben dem Energieverbrauch wird häufig auch der hohe Wasserverbrauch von Beschneiungsanlagen diskutiert (Rixen et al. 2011). Dies gilt insbesondere in trockeneren Gebieten (z. B. inneralpinen Trockenzonen). Auch wenn kein Wasser „verbraucht" wird, sondern am Ende des Winters wieder in den Kreislauf zurückfließt, so könnten lokal durch den hohen Wasserbedarf der Beschneiung in wasserarmen Perioden Nutzungskonflikte entstehen und zu Problemen bei der Schneeproduktion führen. Diesem Problem wurde jedoch großflächig mit dem Bau von Speicheranlagen begegnet. Windverfrachtungen, Sublimation und Verdunstung während und nach der Beschneiung wirken sich negativ auf die Effizienz aus. Diese Wasserverluste sind stark abhängig von lokalen Gegebenheiten und daher nur schwer verallgemeinerbar. Für Österreich wurden die Verluste auf 15-40 \% für Lanzen und auf 5-15\% für Propellerkanonen angegeben (Olefs et al. 2010). In vier französischen Skigebieten war der geringste Verlust $25 \%$ und der höchste über 50 \% (Spandre et al. 2016).
Bei Eissportarten könnte ein Wechsel von Outdoor- zu Indooreinrichtungen zu einem erheblichen steigenden Energieaufwand führen, denn im Mittel liegt der Energiebedarf einer Eishalle um rund das Vierfache höher als für eine Eisfläche im Freien (Lampersberger et al. 2017). Allerdings besteht ein großes Potenzial für energieeffizientere Eishallen, vor allem bei der Kühlanlage, die rund die Hälfte des Strombedarfs ausmacht.

\subsection{Anpassungs-, Minderungsmaßnahmen und Strategien}

Mögliche Anpassungsmaßnahmen der Wintertourismusbranche an den Klimawandel teilen sich in technische Maßnahmen einerseits und eine Anpassung/Diversifizierung der Produktpalette.

Unter technische Anpassungsmaßnahmen fällt die Absicherung des Wintersportbetriebs. Die heute schon weitverbreitete technische Beschneiung (derzeit rund $70 \%$ der Pistenfläche in Österreich; WKO 2019) wird vermutlich noch an Bedeutung hinzugewinnen. Dies beinhaltet die Intensivierung der Beschneiung auf bereits ausgestatteten Flächen (mehr Schneeproduktion in kürzerer Zeit) und die Erhöhung des Anteils der beschneiten Flächen. Auch Langlaufloipen werden zunehmend beschneit, wenn auch hierzu keine österreichweiten Zahlen gefunden werden konnten. Einhergehend mit dieser Entwicklung wird mit einem steigendem Ressourcenbedarf (Wasser- und Energieverbrauch) gerechnet (z. B. Steiger und Stötter 2013). Allerdings zeigen sich auch Bestrebungen hin zu einer Steigerung der Effizienz, sowohl im Bereich der Schneeproduktion als auch beim Schneemanagement. So hat beispielsweise das Forschungsprojekt PROSNOW $^{1}$ zum Ziel, ein Vorhersagesystem zu entwickeln als Grundlage für die Optimierung der Beschneiungspraktiken. Die möglichst effiziente Nutzung der klimatisch gut geeigneten Beschneiungszeiten und Vermeidung von Beschneiung im Grenztemperaturbereich könnten den Energieeinsatz verringern. Kontinuierliches Monitoring der Schneehöhe auf Pistenflächen ermöglicht eine zielgerichtetere Beschneiung und Präparierung. Die hierdurch möglichen Einsparpotenziale werden auf 20-25 \% geschätzt (Rothleitner 2019).

Anlagen, die unabhängig von der Umgebungstemperatur Schnee erzeugen können, sind derzeit entweder noch deutlich weniger energieeffizient als die herkömmliche Beschneiung oder produzieren kleine Eispartikel, die sich einerseits weniger gut für die Präparierung der Skipisten eignen und diese andererseits im Skibetrieb auch nicht so haltbar bleiben (Rothleitner 2019).

Eine weitere technische Maßnahme zur Sicherung der Ressource „Schnee“ ist die Anlage von Schneedepots oder

1 http://prosnow.org/. 
„Snow farming“" sowie das Abdecken von Gletscherflächen. Dies wird eingesetzt, um einen frühen Saisonstart garantieren zu können. Ein Feldversuch in Davos/Schweiz (1620 m) und Martell/Südtirol $(1710 \mathrm{~m})$ ergab eine Konservierung von $72-83 \%$ der ursprünglichen Masse (Grünewald et al. 2018). Die Bilanz des nötigen Energieeinsatzes hierfür im Vergleich zu herkömmlichen Methoden sowie weitere ökologische Probleme, die sich daraus ergeben, sind bisher noch zu wenig untersucht.

Potenziale zur Emissionsreduktion bei Winteraktivitäten bestehen beim Energiemix, d. h. dem Anteil erneuerbarer Energien. Touristische Anbieter können auch eigenen Strom produzieren, z. B. besteht die Möglichkeit, Kraftwerksanlagen in Beschneiungsanlagen zu integrieren (z. B. Kaltenbach, Tirol; Land Tirol 2019). Auch Solaranlagen stellen ein interessantes Potenzial dar, da im Hochgebirge der Wirkungsgrad der Anlagen durch die höhere Strahlung und kühleren Temperaturen höher ist. ${ }^{2}$ Weitere Einsparmöglichkeiten ergeben sich bei der Energieeffizienz von Gebäuden, insbesondere Heizungen und Kühlanlagen (Lampersberger et al. 2017).

Höhenlage und Größe des Skigebiets sind wichtige Determinanten für dessen Rentabilität (Falk und Steiger 2019). Bei repräsentativen Gästebefragungen in Österreich wurde jedoch auch festgestellt, dass die Skigebietsgröße nur ein Faktor von mehreren und auch nicht der wichtigste für die Destinationswahl ist (Pröbstl-Haider und Mostegl 2016; Steiger et al. 2020). Vielmehr ist zu beachten, dass unterschiedliche Segmente auch unterschiedliche Präferenzen haben. Ein Ausweichen in höhere, schneesichere Lagen ist topografisch nur in bestimmten Regionen möglich. Der Klimawandel als Kostentreiber könnte auch den Trend zu größeren Skigebieten weiter verstärken. Beides erhöht den Erschließungsdruck auf sensible Hochgebirgsräume.

Häufig geforderte Anpassungsmaßnahmen sind der Ausbau in Richtung Vierjahreszeitentourismus und Alternativen zu schneeabhängigen Produkten. Ersteres wird seit Jahren von vielen Destinationen und auch Bergbahnen verfolgt. Aufgrund der deutlich höheren Ausgaben von Wintergästen (185€/Tag) im Vergleich zu Sommergästen (160€/Tag; Österreich Werbung 2018e, 2019) ist eine Kompensation von Verlusten im Winter durch mehr Gäste im Sommer nicht ohne Weiteres möglich. Zudem muss berücksichtigt werden, dass die Nutzungsfrequenzen am Berg heute im Sommer deutlich geringer als im Winter sind, eine Erhöhung auf das Winterniveau aber aufgrund unterschiedlicher Nutzungsart ökologisch nicht unproblematisch wäre.

Alternativen zum Thema Schnee im Winter sind derzeit noch rar gesät, vermutlich auch deshalb, weil die verschneite Landschaft auch für Nichtskifahrer einen wichtigen Anreiz für Urlaub in den Alpen darstellt (Bausch und Unseld 2017). Hierbei gilt es auch zu berücksichtigen, dass Investitionen

${ }^{2} \mathrm{http} / / /$ www.ehoch2.co.at/photovoltaik/pitztaler-gletscher.html. in die technische Sicherung des Wintersports durch visuelle und/oder ökologische Beeinträchtigungen in der Destination diese für Nichtwintersportler unattraktiver machen könnten (Bausch et al. 2019).

Ein anderer wichtiger Punkt sind die Nachhaltigkeitsanstrengungen von Wintertourismusdestinationen und -unternehmen. Diese sind nicht nur durch die globale Erwärmung bedroht, sondern agieren auch in umweltsensiblen und geschützten Gebieten und haben im Vergleich zu anderen Branchen oft einen relativ flächen- und ressourcenintensiven Geschäftsbetrieb. Viele Unternehmen und Destinationen sind sich zunehmend ihrer Verantwortung für die Umwelt bewusst und versuchen, den Energieverbrauch zu senken, den Einsatz sauberer Energie zu erhöhen, umweltfreundliche Geschäftspraktiken und Verkehrsmittel am Zielort zu nutzen sowie Maßnahmen zum Schutz des Bodens zu ergreifen. Auch grüne Zertifizierungskennzeichen (Umweltzertifizierungsstandards wie die ISO-14000er-Serie oder eine Zertifizierung nach dem Eco-Management and Audit Scheme - kurz EMAS -, wie zum Beispiel bei der Schmittenhöhebahn in Zell am See) und die Erstellung von Nachhaltigkeitsberichten (Global Reporting Initiative, GRI) zählen zu den möglichen Maßnahmen (Österreich Werbung 2019). Bei den Skibetrieben gibt es hierbei noch deutlichen Nachholbedarf. Wenige Skiliftunternehmen (wie Aspen Ski Resort in den USA, Compagnie des Alpes in Frankreich, Alpe d'Huez, Zermatt Bergbahnen in der Schweiz sowie Betriebe in Lech, Planai-Hochwurzen und Kaprun in Österreich) haben erfolgreich eine Umweltzertifizierung beantragt (ISO 14000; Quelle: ISO-Datenbank).

Für Bemühungen in Richtung klimaneutraler Skigebiete gibt es erste Belege. ${ }^{3}$ Dies wurde z. B. durch den Bezug bzw. die Eigenproduktion des gesamten Stroms aus erneuerbaren Energieträgern, Wiederaufforstung in der eigenen Region sowie die Kompensation der noch anfallenden $\mathrm{CO}_{2}$-Emissionen erreicht. Dies ermöglichte eine $\mathrm{CO}_{2}$-Bilanzierung, welche auch als Grundlage für Verbesserungsmaßnahmen innerhalb des Unternehmens dienen soll.

\subsection{Handlungsoptionen, Kommunikations- und Forschungsbedarf}

Klimawandel bedeutet nicht zwangsläufig das Ende von schneebasiertem Wintertourismus. Die zu erwartenden Veränderungen sind regional sehr unterschiedlich ebenso wie die verfügbaren Anpassungsmaßnahmen. Die Beurteilung von Klimarisiken ist daher ein wichtiger Teil der Risikoabschät-

\footnotetext{
${ }^{3}$ Siehe die Brunni-Bahnen (http://www.brunni.ch/ueber-uns-links/ ueber-uns/umwelterklaerung/), das seit 2018 erste als klimaneutral zertifizierte Bergbahnunternehmen der Schweiz, oder die Silvrettaseilbahn AG (https://www.ischgl.com/de/More/Seilbahnunternehmen/Klimaneutrales-Skigebiet), die ebenfalls seit 2019 als klimaneutral zertifiziert wurde.
} 
zung von Unternehmen im Tourismus. Trotz gegebener Unsicherheiten in der Klimamodellierung können bestehende Modelle einen wertvollen Beitrag zur Risikoanalyse liefern. Jüngere Entwicklungen im Bereich des Schneemanagements sind wissenschaftlich noch unzureichend erforscht. Für eine solide Abschätzung der Effizienz, Wirksamkeit und der Folgewirkungen ist noch mehr Forschung gefordert.

Auf Nachfrageseite bestehen ebenfalls noch Wissenslücken: Wo existieren Kipppunkte, die das Verhalten der Gäste nachhaltig beeinflussen, sei es die Destinationswahl oder auch die Entscheidung, keinen Wintersport mehr auszuüben? Grundsätzlich sind auch weitere externe Faktoren, die das Tourismussystem beeinflussen, mit zu berücksichtigen, da sich diese Faktoren gegenseitig verstärken oder auch abschwächen können. So wurden z. B. die Folgen des demografischen Wandels im Quellmarkt Deutschland für den Skitourismus in Tirol bis zur Mitte des Jahrhunderts als bedeutender eingestuft als der Klimawandel (Steiger 2012). Forschungsbedarf ist auch in Bezug auf schneeunabhängige Urlaubsangebote und -produkte gegeben. Wie steht es beispielsweise um die Schneesensitivität von Gästen, die keinen klassischen Schneesport betreiben? Welche Produkte könnten Gäste ansprechen, für die Winterurlaub in den Alpen bisher keine Rolle spielt? Welche Destinationstypen wären für solch „neue Wintergäste“ attraktiver? Gut erschlossene Wintersportdestinationen oder im Winter noch weniger erschlossene Tourismusregionen?

Empirische Analysen der finanziellen Situation von Hotels und Seilbahnen in Gebieten, die besonders vom Klimawandel betroffen sind, sind ebenfalls notwendig. Dies erfordert den Zugriff auf Betriebsdaten der amtlichen Statistik, die mit Geodaten verknüpft werden können. Zudem wäre eine Betrachtung der unterschiedlichen Wertschöpfung von Sommerund Wintertourismus, inklusive der unterschiedlichen Wertschöpfungsabflüsse empfehlenswert.

Im Bereich der Mitigation besteht großer Forschungsbedarf bei der $\mathrm{CO}_{2}$-Bilanz des Wintertourismus. Bisherige Literatur für Österreich ist entweder nur auf einen Teilbereich beschränkt, veraltet und/oder beruht auf einer Vielzahl von Annahmen aufgrund fehlender Daten. Für effektive Klimaschutzmaßnahmen im Wintertourismus sind ein solider Status quo sowie Monitoring der Bilanz und Wirksamkeit von eingeführten Maßnahmen nötig.

In den meisten jüngeren Impact-Studien werden die RCPSzenarien 4.5 und 8.5 verwendet. Das gemäßigte Szenario RCP 2.6, bei welchem eine große Wahrscheinlichkeit zur Erreichung der Paris-Klimaziele besteht, fand bisher noch keine Anwendung. Es ist anzunehmen, dass die Folgen für den Wintertourismus im RCP-2.6-Szenario geringer ausfallen würden. Die Bandbreite der Folgen in unterschiedlichen RCP-Szenarien aufzuzeigen, könnte somit ein gewichtiges Argument für rasche und tief greifende Klimaschutzmaßnahmen darstellen.
Politik und Wirtschaft müssen sich mit den zunehmenden Leistungsunterschieden zwischen begünstigten und nichtbegünstigten Gebieten befassen. Eine stärkere öffentliche Unterstützung für benachteiligte Gebiete ist kostspielig und kann Anpassungsprobleme nur kurzfristig lindern. Im Allgemeinen sollten Fördermaßnahmen (z. B. öffentliche Investitionszuschüsse) evaluiert werden.

\subsection{Zusammenfassung}

Das hohe Niveau der touristischen Kennzahlen im Winter konnte in den letzten Jahren gehalten werden. Allerdings zeigen Untersuchungen, dass im Hauptherkunftsmarkt Deutschland das Interesse am Skifahren abnimmt, während z. B. die Beliebtheit des Winterwanderns zunimmt (hohe Übereinstimmung, mittlere Beweislage). Dadurch ergibt sich ein steigender Wettbewerbsdruck, der die Rahmenbedingungen für manche Skigebiete und Destinationen erschweren dürfte. Der Klimawandel verändert die Bedingungen für natürlichen Schneefall und Beschneiung und das Risiko von schwierigen Situationen vor allem in den Saisonrandzeiten, aber in weiterer Folge auch im Hochwinter (hohe Übereinstimmung, starke Beweislage). Dies verschärft weiter den Wettbewerbsdruck und könnte zu einem Verdrängungswettbewerb führen.

Pauschale Aussagen zur Zukunft des Wintertourismus sind nicht möglich, vielmehr wird die Zukunft aus einem komplexen Mix aus Gewinnern und Verlierern der Entwicklung bestehen. Skifahren wird technisch gesehen auch Ende des 21. Jahrhunderts noch möglich sein, nur vermutlich nicht mehr überall dort, wo das heute noch der Fall ist (hohe Übereinstimmung, starke Beweislage). Untersuchungen zufolge könnten Mitte des Jahrhunderts noch 52-72 \% (je nach Entwicklung der Treibhausgasemissionen) der Skigebiete in Österreich als schneesicher eingestuft werden, bei heutiger Beschneiungstechnologie und -kapazität. Die sich daraus ergebenden Auswirkungen auf die Nachfrage werden auf nationaler Ebene als moderat eingeschätzt $(<10 \%)$, da nach wie vor noch ausreichend viele Skigebiete schneesicher sind. Allerdings könnten sich große räumliche Nachfrageverschiebungen von $\pm 50 \%$ ergeben mit entsprechenden Folgen für die betroffenen Destinationen und Betriebe (hohe Übereinstimmung, schwache Beweislage). Die Herausforderung wird künftig sein, derartige Änderungen, seien sie positiv oder negativ, zu managen. Dazu zählt nicht nur die Entwicklung von schneeunabhängigen Winterprodukten, sondern auch die Absicherung des Wintersports in Regionen, die klimatisch begünstigt sind.

Der Wintertourismus trägt auch selbst zum Klimawandel bei. Daten für eine genauere Abschätzung des $\mathrm{CO}_{2}$-Beitrags des Wintertourismus in Österreich sind derzeit nicht verfügbar. Die wenigen Belege zeigen allerdings, dass Verkehr und Beherbergung den mit Abstand höchsten Anteil an den 
touristischen Emissionen tragen (hohe Übereinstimmung, schwache Beweislage). Hier besteht der größte Handlungsbedarf, um den touristischen Anteil der Treibhausgasemissionen im Wintertourismus zu reduzieren. Bemühungen der Skigebietsbetreiber, ihre Emissionen zu reduzieren, z. B. durch effizienteres Schnee- und Pistenmanagement oder auch Energieerzeugung aus regenerativen Quellen, sind in diesem Zusammenhang zu begrüßen, können aber nur einen kleinen Beitrag zur Erreichung der Klimaschutzziele leisten.

\section{Kernaussagen - Kapitel 6}

- Der Klimawandel verschärft den ohnehin steigenden Wettbewerbsdruck. Kürzere Saisonen, ein steigendes Risiko von Ausfalltagen aufgrund von Schneemangel sowie der steigende Beschneiungsbedarf stellen die Skigebiete vor Herausforderungen (hohe Übereinstimmung, starke Beweislage).

- Skifahren wird in Österreich bei entsprechender Beschneiung auch weiterhin möglich sein, jedoch ist mit einer Konzentration der Nachfrage auf Gunstlagen sowie steigenden Kosten zu rechnen (hohe Übereinstimmung, starke Beweislage).

- Die Gesamtnachfrage nach Wintertourismus in Österreich erscheint trotz Klimafolgen bis Mitte des Jahrhunderts auf nationaler Ebene stabil, allerdings mit großen räumlichen Umverteilungen innerhalb Österreichs. Dies schließt Veränderungen bei den Anteilen der Skifahrerinnen und Skifahrer nicht aus (hohe Übereinstimmung, mittlere Beweislage).

- $\mathrm{CO}_{2}$-Einsparpotenziale bei Skigebieten bestehen durch den Bezug erneuerbarer Energie, durch kontinuierliches Monitoring der Schneehöhen auf den Pisten, emissionsärmere Fahrzeuge sowie bei der Verbesserung der Energieeffizienz von Gebäuden. Bei Aktivitäten abseits des alpinen Skilaufs stellen die Umstellung auf erneuerbare Energien sowie energieeffizientere Gebäude Potenziale zur Emissionsreduktion dar.

- Forschungsbedarf besteht vor allem bei den Treibhausgasemissionen des Tourismus und bei den möglichen Gästereaktionen auf wärmer werdende Winter und den damit verbundenen Anpassungsmaßnahmen der Anbieterseite.

\section{Literatur}

Abegg, B., König, U. \& Maisch, M. (1994) Klimaänderung und Gletscherskitourismus. Geographica Helvetica 49(3), 103-114. DOI: https://doi.org/10.5194/gh-49-103-1994

Abegg, B. (1996) Klimaänderung und Tourismus. Klimafolgenforschung am Beispiel des Wintertourismus in den Schweizer Alpen. Vdf Zürich, Zürich, Schweiz.
Abegg, B., Agrawala, S., Crick, F. \& de Montfalcon, A. (2007) Climate change impacts and adaptation in winter tourism. In: Agrawala, S. (Hrsg.) Climate Change in the European Alps. Adapting Winter Tourism and Natural Hazards Management, S. 25-60. OECD, Paris, Frankreich.

Bätzing, W. (2017) Orte guten Lebens.: Visionen für einen Alpentourismus zwischen Wildnis und Freizeitpark. In: Luger, K. \& Rest, F. (Hrsg.) Alpenreisen: Erlebnis, Raumtransformationen, Imagination, S. 215-236. StudienVerlag, Innsbruck, Österreich.

Bausch, T. \& Unseld, C. (2017) Winter tourism in Germany is much more than skiing! Consumer motives and implications to Alpine destination marketing. Journal of Vacation Marketing 24(3), 203-217. DOI: https://doi.org/10.1177/1356766717691806

Bausch, T., Humpe, A. \& Gössling, S. (2019) Does climate change influence guest loyalty at Alpine winter destinations? Sustainability 11(15), 4233. DOI:https://doi.org/10.3390/su11154233

BMVI (2018) Verkehr in Zahlen 2018/2019. Bundesministerium für Verkehr und digitale Infrastruktur (BMVI), Kraftfahrt-Bundesamt, Flensburg, Deutschland. Online unter: https://www.bmvi.de/SharedDocs/DE/Publikationen/G/verkehr-in-zahlen_2018-pdf.html (letzter Zugriff: 05.05.2020).

Breiling, M., Charamza, P. \& Skage, O.R. (1997) Klimasensibilität österreichischer Bezirke mit besonderer Berücksichtigung des Wintertourismus. Institut für Landschaftsplanung Alnarp, Schwedische Universität für Agrarwissenschaften, Alnarp, Schweden. Online unter: http://www.breiling.org/publ/klimwt.pdf (letzter Zugriff: 05.05.2020).

Butler, R.W. (1980) The concept of a tourist area cycle of evolution: implications for management of resources. The Canadian Geographer/Le Géographe canadién 24(1), 5-12. DOI: https://doi.org/ 10.1111/j.1541-0064.1980.tb00970.x

Chimani, B., Heinrich, G., Hofstätter, M., Kerschbaumer, M., Kienberger, S., Leuprecht, A., Lexer, A., Peßenteiner, S., Poetsch, M.S., Salzmann, M., Spiekermann, R., Switanek, M. \& Truhetz, H. (2016) ÖKS15 - Klimaszenarien für Österreich. Daten, Methoden und Klimaanalyse. Projektendbericht. Online unter: https://data.ccca.ac.at/ dataset/a4ec86ca-eeae-4457-b0c7-78eed6b71c05 (letzter Zugriff: 01.04.2020).

Damm, A., Köberl, J. \& Prettenthaler, F. (2014) Does artificial snow production pay under future climate conditions? - A case study for a vulnerable ski area in Austria. Tourism Management 43, 8-21. DOI: https://doi.org/10.1016/j.tourman.2014.01.009

Damm, A., Greuell, W., Landgren, O. \& Prettenthaler, F. (2017) Impacts of $+2{ }^{\circ} \mathrm{C}$ global warming on winter tourism demand in Europe. Climate Services 7, 31-46. DOI: https://doi.org/10.1016/j. cliser.2016.07.003

Diolaiuti G., Smiraglia, C., Pelfini, M., Belò, M., Pavan, M. \& Vassena, G. (2006) The recent evolution of an Alpine glacier used for summer skiing (Vedretta Piana, Stelvio Pass, Italy). Cold Regions Science and Technology 44(3), 206-216. DOI: https://doi.org/10.1016/j.coldregions.2005.11.006

DSV (2019) Technischer Schnee \& Pisten. Deutscher Skiverband. Deutscher Skiverband (DSV), Planegg, Deutschland. Online unter: https://www.deutscherskiverband.de/ueber_uns_umwelt_fragen techn_de.html (letzter Zugriff: 01.04.2020).

Duprez, $\bar{C} . \&$ Burget, L. (2007) Bilan gaz a effet de serre en station Saint Martin de Belleville (Les Menuires, Val Thorens). Studie im Auftrag Agence De l'Environnement et de la Maîtrise de l'Energie (ADEME) und der Gemeinde Saint Martin de Belleville. Online unter: https:// www.yumpu.com/fr/document/view/30300376/bilan-gaz-a-effet-deserre-en-station-mountain-riders (letzter Zugriff: 13.12.2018).

E-Control (2018) Pro-Kopf-Verbrauch: Entwicklung des Inlandstromverbrauchs sowie des Haushaltsstromverbrauchs je Einwohner. E-Control, Wien, Österreich. Online unter: https://www.e-control. at/statistik/strom/betriebsstatistik/jahresreihen (letzter Zugriff: 05.05.2020). 
Falk, M. (2010) A dynamic panel data analysis of snow depth and winter tourism. Tourism Management 31(6), 912-924. DOI: https://doi.org/ 10.1016/j.tourman.2009.11.010

Falk, M. (2013a) A survival analysis of ski lift companies. Tourism Management 36, 377-390. DOI: https://doi.org/10.1016/j.tourman.2012.10.005

Falk, M. (2013b) Impact of long-term weather on domestic and foreign winter tourism demand. International Journal of Tourism Research 15(1), 1-17. DOI: https://doi.org/10.1002/jtr.865

Falk, M. (2017) Gains from horizontal collaboration among ski areas. Tourism Management 60, 92-104. DOI: https://doi.org/10.1016/j. tourman.2016.11.008

Falk, M. \& Hagsten, E. (2018) Winter weather anomalies and individual destination choice. Sustainability 10(8), 2630. DOI: https://doi. org $/ 10.3390 / \mathrm{su} 10082630$

Falk, M. \& Lin, X. (2018) Sensitivity of winter tourism to temperature increases over the last decades. Economic Modelling 71, 174-183. DOI: https://doi.org/10.1016/j.econmod.2017.12.011

Falk, M. \& Steiger, R. (2018) An exploration of the debt ratio of ski lift operators. Sustainability 10(9), 2985. DOI: https://doi.org/10.3390/ su10092985

Falk, M. \& Steiger, R. (2019) Size facilitates profitable ski lift operations. Tourism Economics. DOI: https://doi. org $/ 10.1177 / 1354816619868117$

Firgo, M. \& Fritz, O. (2017) Does having the right visitor mix do the job? Applying an econometric shift-share model to regional tourism developments. The Annals of Regional Science 58(3), 469-490. DOI: https://doi.org/10.1007/s00168-016-0803-4

Fleischhacker, E., Formayer, H., Seisser, O., Wolf-Eberl, S. \& KrompKolb, H. (2009) Auswirkungen des Klimawandels auf das künftige Reiseverhalten im österreichischen Tourismus am Beispiel einer repräsentativen Befragung der österreichischen Urlaubsreisenden. BOKU-Met Report 19. Im Auftrag des Bundesministeriums für Wirtschaft, Familie und Jugend (BMWFJ), Wien, Österreich. Online unter: https://meteo.boku.ac.at/report/ (letzter Zugriff: 05.05.2020).

Fleischhacker, V. (2018) Klimawandel und Wintersporttourismus in Österreich 2030. Institut für touristische Raumplanung-ITR, Tulln an der Donau, Österreich. Online unter: http://www.tourismusforschungaustria.at/440348425 (letzter Zugriff: 05.05.2020).

Friesenbichler, J. (2003) Energieeinsatz und $\mathrm{CO}_{2}$-Emissionen im Wintertourismus. Diplomarbeit im Rahmen des Fachhochschulstudiengangs „Infrastrukturwirtschaft“ der FH Joanneum Kapfenberg, Österreich.

Gössling, S. \& Hall, M.C. (2006) Uncertainties in predicting tourist flows under scenarios of climate change. Climatic Change 79(3-4), 163-173. DOI: https://doi.org/10.1007/s10584-006-9081-y

Gössling, S., Scott, D. \& Hall, C.M. (2018) Global trends in length of stay: implications for destination management and climate change. Journal of Sustainable Tourism, 26(12), 2087-2101. DOI: https:// doi.org/10.1080/09669582.2018.1529771

Grünewald, T., Wolfsperger, F. \& Lehning, M. (2018) Snow farming: conserving snow over the summer season. The Cryosphere 12(1), 385-400. DOI: https://doi.org/10.5194/tc-12-385-2018

Köberl, J., Prettenthaler, F., Nabernegg, S. \& Schinko, T. (2015) Tourism. In: Steininger, K., König, M., Bednar-Friedl, B., Kranzl, L., Loibl, W. \& Prettenthaler, F. (Hrsg.) Economic evaluation of climate change impacts: development of a cross-sectoral framework and results for Austria, S. 367-388. Springer, Cham, Schweiz.

Lampersberger, P., Benke, G., Grim, M., Hüttler, W., Preßmair, G., Schwarz-Viechtbauer, K. \& Szeywerth, F. (2017) EnergieFit: Innovative Energietechnologien für Sportstätten. Teil 1: Leitfaden für Entscheidungsträger. Gefördert durch den Klima- und Energiefonds im Rahmen des Programms „Energieforschungsprogramm“. Online unter: https://www.klimafonds.gv.at/wp-content/uploads/ sites/6/Leitfaden-EntscheidungstrgerEnergieFit.pdf (letzter Zugriff:02.06.2020).
Land Tirol (2019) Wasserbuch - Auszug des Landes Tirol: 9/1980 Beschneiungsanlage Hochzillertal \& Retentionsmaßnahmen Aschauerbach. Amt der Tiroler Landesregierung, Innsbruck, Österreich. Online unter: https://portal.tirol.gv.at/wisSrvPublic/wis/wbo_wb auszug.aspx?TYPE=T\&ANL_ID=T60929093 (letzter Zugriff: 04.06.2020).

Landauer, M. \& Pröbstl, U. (2008) Klimawandel, Skilanglauf und Tourismus in Österreich. Wahrnehmung durch Skilangläufer, Landschaftserlebnis und mögliche Adaptionsstrategien. Naturschutz und Landschaftsplanung 40(10), 336-342. Online unter: https://www. nul-online.de/artikel.dll/NuL-08-10-S336-342_MTMxODM0Mw. PDF (letzter Zugriff: 06.05.2020)

Landauer, M., Pröbstl, U. \& Haider, W. (2012) Managing cross-country skiing destinations under the conditions of climate change - Scenarios for destinations in Austria and Finland. Tourism Management 33(4), 741-751. DOI: https://doi.org/10.1016/j.tourman.2011.08.007

Landauer, M., Haider, W. \& Pröbstl-Haider, U. (2013) The Influence of Culture on Climate Change Adaptation Strategies: preferences of cross-country skiers in Austria and Finland. Journal of Travel Research 53(1), 96-110. DOI: https://doi. org/10.1177/0047287513481276

Luthe, T. (2009) SkiSustain: vulnerability to global change and sustainable adaptation of ski tourism. Institut für Outdoor Sport und Umweltforschung, Deutsche Sportuniversität Köln, Deutschland. Online unter: https://www.dshs-koeln.de/fileadmin/redaktion/Institute/Natursport und Oekologie/Veroeffentlichungen/Schriftenreihe/INOEK_Band_25_Dissertation_Luthe.pdf (letzter Zugriff: 06.05.2020).

Mayer, M., Steiger, R. \& Trawöger, L. (2007) Technischer Schnee rieselt vom touristischen Machbarkeitshimmel - Schneesicherheit und technische Beschneiung in westösterreichischen Skidestinationen vor dem Hintergrund klimatischer Wandlungsprozesse. In: Österreichische Geographische Gesellschaft (Hrsg.) Mitteilungen der Österreichischen Geographischen Gesellschaft Band 149/2007, S. 157-180. Österreichische Geographische Gesellschaft, Wien, Österreich.

Mayer, M., Demiroglu, O.C. \& Ozcelebi, O. (2018) Microclimatic volatility and elasticity of glacier skiing demand. Sustainability 10(19), 3536. DOI: https://doi.org/10.3390/su10103536

Olefs M., Fischer, A. \& Lang, J. (2010) Boundary conditions for artificial snow production in the Austrian Alps. Journal of Applied Meteorology \& Climatology 49(6), 1096-1113. DOI: https://doi.org/ 10.1175/2010JAMC2251.1

Österreich Werbung (2012) T-MONA Urlauber Winter 2011/12. Reiseverhalten der Gäste in Österreich. Zur Verfügung gestellt von Österreich Werbung, Wien, Österreich.

Österreich Werbung (2018a) Österreich-Urlauber im Winter 2017/18. Zur Verfügung gestellt von Österreich Werbung, Wien, Österreich..

Österreich Werbung (2018b) Winterurlauber in Österreich. Langläufer. Zur Verfügung gestellt von Österreich Werbung, Wien, Österreich. Online unter: https://www.austriatourism.com/tourismusforschung/ studien-und-berichte/langlaeufer-winter-201718/ (letzter Zugriff: 07.05.2020).

Österreich Werbung (2018c) Winterurlauber in Österreich. Skifahrer. Zur Verfügung gestellt von Österreich Werbung, Wien, Österreich.

Österreich Werbung (2018d) Winterurlauber in Österreich. Snowboarder. Zur Verfügung gestellt von Österreich Werbung, Wien, Österreich.

Österreich Werbung (2018e) Ausgaben der Gäste in Österreich. T-MONA Urlauberbefragung 2018. Zur Verfügung gestellt von Österreich Werbung, Wien, Österreich.

Österreich Werbung (2019) Ausgaben der Gäste in Österreich. T-MONA Urlauberbefragung 2019. Zur Verfügung gestellt von Österreich Werbung, Wien, Österreich.

Prettenthaler F. \& Formayer, H. (Hrsg.) (2011) Tourismus im Klimawandel: Zur regionalwirtschaftlichen Bedeutung des Klimawandels für die österreichischen Tourismusgemeinden. Studien zum Klima- 
wandel in Österreich, Band 6. Verlag der Österreichischen Akademie der Wissenschaften (ÖAW), Wien, Österreich.

Prettenthaler, F., Formayer, H., Aumayer, C., Haas, P., Habsburg-Lothringen, C., Hofstätter, M., Richter, V. \& Vetters, N. (2009) Global change impact on tourism: der sozioökonomische Einfluss des Klimawandels auf den Winter- und Sommertourismus in Österreich. Joanneum Research Forschungsgesellschaft, Institut für Technologieund Regionalpolitik und Universität für Bodenkultur Wien, Institut für Meteorologie. Zitiert in: APCC (2014) Österreichischer Sachstandsbericht Klimawandel 2014 (AAR14), S. 670. Austrian Panel on Climate Change (APCC), Verlag der Österreichischen Akademie der Wissenschaften (ÖAW), Wien, Österreich. Online unter: http://www. austriaca.at/APCC_AAR2014.pdf (letzter Zugriff: 02.06.2020).

Pröbstl, U. (2006) Kunstschnee und Umwelt: Entwicklung und Auswirkungen der technischen Beschneiung. Haupt, Bern u. a., Schweiz.

Pröbstl-Haider, U. \& Haider, W. (2013) Tools for measuring the intention for adapting to climate change by winter tourists: some thoughts on consumer behavior research and an empirical example. Tourism Review 68(2), 44-55. DOI: https://doi.org/10.1108/TR-04-2013-0015

Pröbstl-Haider, U., Mostegl, N. \& Haider, W. (2015) Einfluss von Skigebietsverbindungen im Bereich Stubai/westliches Mittelgebirge auf die regionale und deutsche Nachfrage durch Wintersportler. Endbericht. Online unter: https://www.brueckenschlag-tirol.com/app/download/12644911327/Endbericht+Arge+Br\%C3\%BCckenschlag+FINAL+2015.pdf?t=1473671355 (letzter Zugriff: 09.05.2020).

Pröbstl-Haider, U. \& Mostegl, N. (2016) Skigebiete im Vergleich: macht Größe allein schon sexy? FdSnow 49, 28-35.

Pröbstl-Haider, U. \& Flaig, R. (2019) The knockout deal - pricing strategies in Alpine ski resorts. In: Pröbstl-Haider, U., Richins, H. \& Türk, S. (Hrsg.) Winter tourism: trends and challenges, S. 116-137. CABI, Wallingford, Vereinigtes Königreich.

Pröbstl-Haider, U. \& Mostegl, N. (2019) A matter of culture: how cultural differences shape skiing motivation, behaviour and destination choice. In: Pröbstl-Haider, U., Richins, H. \& Türk, S. (Hrsg.) Winter tourism: trends and challenges, S. 192-211. CABI, Wallingford, Vereinigtes Königreich.

Rixen C., Teich, M., Lardelli, C., Gallati, D., Pohl, M., Pütz, M. \& Bebi, P. (2011) Winter tourism and climate change in the Alps: an assessment of resource consumption, snow reliability, and future snowmaking potential. Mountain Research and Development 31(3), 229 236. DOI: https://doi.org/10.1659/MRD-JOURNAL-D-10-00112.1

Roth, R., Krämer, A. \& Görtz, M. (2012) Grundlagenstudie Wintersport Deutschland. Schriftenreihe Natursport und Ökologie, Band 26. Institut für Natursport und Ökologie, Deutsche Sporthochschule Köln (DSHS), Deutschland.

Roth, R., Krämer, A. \& Severiens, J. (2018) Zweite Nationale Grundlagenstudie Wintersport Deutschland 2018. Schriftenreihe Stiftung Sicherheit im Skisport (SIS), Planegg, Deutschland. Online unter: https://www.stiftung.ski/sis-lab/grundlagenstudie-wintersport/ (letzter Zugriff: 12.05.2020).

Rothleitner, M. (2019) Persönliche Auskunft Michael Rothleitner, Schneezentrum Tirol, Österreich.

Rutty, M., Scott, D., Johnson, P., Jover, E., Pons, M. \& Steiger, R. (2015a) Behavioural adaptation of skiers to climatic variability and change in Ontario, Canada. Journal of Outdoor Recreation and Tourism 11, 13-21. DOI: https://doi.org/10.1016/j.jort.2015.07.002

Rutty, M., Scott, D., Johnson, P., Jover, E., Pons, M. \& Steiger, R. (2015b) The geography of skier adaptation to adverse conditions in the Ontario ski market. The Canadian Geographer/Le Géographe canadién 59(4), 391-403. DOI: https://doi.org/10.1111/cag.12220

Schwaiger, H., Bird, D.N., Damm, A., Kortschak, D. \& Prettenthaler, F. (2017) Die Klima- und Energiebilanz von Skigebieten mit technischer Beschneiung unter Berücksichtigung des Albedo-Effektes. Joanneum Research Forschungsgesellschaft mbH, LIFE - Zentrum für Klima, Energie und Gesellschaft, Graz, Österreich. Online unter: https://www.joanneum.at/fileadmin/LIFE/News_Bilder_Logos/
news/Klima-und_Energiebilanz_von_Beschneiung.pdf (letzter Zugriff: 15.04 .2020$)$.

Spandre P., Morin, S., Lafaysse, M., Lejeune, Y., François, H. \& George-Marcelpoil, E. (2016) Integration of snow management processes into a detailed snowpack model. Cold Regions Science and Technology 125, 48-64. DOI: https://doi.org/10.1016/j.coldregions.2016.01.002

Statistik Austria (2019) Tourismus. Bundesanstalt Statistik Österreich, Wien, Österreich. Online unter: https://www.statistik.at/ web_de/statistiken/wirtschaft/tourismus/index.html (letzter Zugriff: 07.05.2020).

Steiger, R. \& Mayer, M. (2008) Snowmaking and climate change: future options for snow production in Tyrolean ski resorts. Mountain Research and Development 28(3), 292-298. DOI: https://doi. org/10.1659/mrd.0978

Steiger, R. (2010) The impact of climate change on ski season length and snowmaking requirements. Climate Research 43(3), 251-262. DOI: https://doi.org/10.3354/cr00941

Steiger, R. (2011) The impact of snow scarcity on ski tourism: an analysis of the record warm season 2006/07 in Tyrol (Austria). Tourism Review, 66(3), 4-15. DOI: https://doi.org/10.1108/16605371111175285

Steiger, R. (2012) Scenarios for skiing tourism in Austria: integrating demographics with an analysis of climate change. Journal of Sustainable Tourism 20(6), 867-882. DOI: https://doi.org/10.1080/096 69582.2012 .680464

Steiger, R. \& Abegg, B. (2013) The sensitivity of Austrian ski areas to climate change. Tourism Planning \& Development 10(4), 480-493. DOI: https://doi.org/10.1080/21568316.2013.804431

Steiger, R. \& Stötter, J. (2013) Climate change impact assessment of ski tourism in Tyrol. Tourism Geographies 15(4), 577-600. DOI: https:// doi.org/10.1080/14616688.2012.762539

Steiger, R. \& Abegg, B. (2015) Klimawandel und Konkurrenzfähigkeit der Skigebiete in den Ostalpen. In: Egger, R. \& Luger, K. (Hrsg.) Tourismus und mobile Freizeit - Lebensformen, Trends, Herausforderungen, S. 319-332. Books on Demand, Norderstedt, Deutschland.

Steiger, R. \& Posch, E. (2017) Zusammenfassung der Umfrageergebnisse 2015/2016. ,Wie reagieren unsere Wintergäste in schneearmen Saisonperioden? " Projekt CCSBD-AT. Gefördert durch den Klimaund Energiefonds im Rahmen des Programms „Austrian Climate Research Programme“ (ACRP). Online unter: https://geographie. uibk.ac.at/blog/atg/wp-content/uploads/sites/4/2017/11/Endbericht Befragung_Juni2017_Web.pdf (letzter Zugriff: 07.05.2020).

Steiger, R., Posch, E., Pons-Pons, M. \& Vilella, M. (2018) Climate change impacts on skier behaviour and spatial distribution of skiers in Austria. Publizierbarer Endbericht zum Projekt CCSBD-AT, gefördert durch den Klima- und Energiefonds im Rahmen des Programms „Austrian Climate Research Programme“ (ACRP). Online unter: https://www.klimafonds.gv.at/wp-content/uploads/sites/6/12EB-CCSBD-AT-.pdf (letzter Zugriff: 07.05.2020).

Steiger, R., Scott, D., Abegg, B., Pons, M. \& Aall, C. (2019) A critical review of climate change risk for ski tourism. Current Issues in Tourism 22(11), 1343-1379. DOI: https://doi.org/10.1080/136835 00.2017.1410110

Steiger, R., Posch, E., Tappeiner, G. \& Walde, J. (2020) The impact of climate change on demand of ski tourism: a simulation study based on stated preferences. Ecological Economics 170, 106589. DOI: https://doi.org/10.1016/j.ecolecon.2019.106589

Steiger, R. \& Scott, D. (2020) Ski tourism in a warmer world: increased adaptation and regional economic impacts in Austria. Tourism Management 77, 104032. DOI: https://doi.org/10.1016/j.tourman.2019.104032

Töglhofer, C., Eigner, F. \& Prettenthaler, F. (2011) Impacts of snow conditions on tourism demand in Austrian ski areas. Climate Research 46(1), 1-14. DOI: https://doi.org/10.3354/cr00939

Umweltbundesamt (2018) Vergleichende Treibhausgasbilanz typischer Arten von Urlauben. Studie im Auftrag der Wirtschaftskammer Ös- 
terreich (WKO), Fachverband Seilbahnen; in Zusammenarbeit mit Wintersport-Tirol. Umweltbundesamt $\mathrm{GmbH}$, Wien, Österreich.

Unbehaun, W., Pröbstl, U. \& Haider, W. (2008) Trends in winter sport tourism: challenges for the future. Tourism Review 63(1), 36-47. DOI: https://doi.org/10.1108/16605370810861035

Vanat, L. (2018) 2018 International Report on Snow \& Mountain Tourism. Laurent Vanat, Genf, Schweiz.

Weihs, P. \& Laimighofer, J. (2019) SnowAlb - Effekte künstlicher Beschneiung auf den Strahlungshaushalt der Skiregion Saalbach-Hinterglemm. Endbericht von Start-Clim2018.C in StartClim2018: Synergien und Nutzungskonflikte bei der Umsetzung von Klimawandelanpassung und den Sustainable Development Goals in Österreich aus Sicht der Klimaforschung. Online unter: http://www.startclim. at/fileadmin/user_upload/StartClim2018_reports/StCl18C_lang.pdf (letzter Zugriff: 16.04.2020).

WKO (2001) Berichtsblätter. Wirtschaftsbericht der Seilbahnen. Bilanzjahr Winter 2000/2001 - Sommer 2001. Wirtschaftskammer Österreich (WKO), Fachverband Seilbahnen, Wien, Österreich

WKO (2007) Berichtsblätter. Trendmonitor Saisonbilanz Winter 2006/2007. Wirtschaftskammer Österreich (WKO), Fachverband Seilbahnen, Wien, Österreich

WKO (2018) Seilbahnen: Zahlen/Daten/Fakten. Wirtschaftskammer Österreich (WKO), Wien, Österreich. Online unter: https://www.
wko.at/branchen/transport-verkehr/seilbahnen/ZahlenDatenFakten. html (letzter Zugriff: 07.05.2020).

WKO (2019) FACTSHEET - Technische Beschneiung in Österreich. Wirtschaftskammer Österreich (WKO), Fachverband Seilbahnen, Wien, Österreich. Online unter: https://www.wko.at/branchen/transport-verkehr/seilbahnen/factsheet-beschneiung.pdf (letzter Zugriff: 07.05.2020)

Zegg, R., Küng, T. \& Grossrieder, R. (2010) Energiemanagement Bergbahnen. Seilbahnen Schweiz (SBS), Bern und Chur, Schweiz.

Zellmann, P. \& Mayrhofer, S. (2010) So sportlich ist Österreich: die Sportausübung im Europa- und Bundesländer-Vergleich. ift Forschungstelegramm 12/2010. IFT Institut für Freizeit- und Tourismusforschung, Wien, Österreich. Online unter: https://www.freizeitforschung.at/data/forschungsarchiv.html (letzter Zugriff: 07.05.2020).

Zellmann, P. \& Mayrhofer, S. (2018a) Skifahren ist teurer geworden, wie vieles andere auch. ift Forschungstelegramm 7/2018. IFT Institut für Freizeit- und Tourismusforschung, Wien, Österreich. Online unter: http://www.freizeitforschung.at/data/forschungsarchiv.html (letzter Zugriff: 12.05.2020).

Zellmann, P. \& Mayrhofer, S. (2018b) Sportmonitor 2018: Österreich wird sportlicher. ift Forschungstelegramm 3/2018. IFT Institut für Freizeit- und Tourismusforschung, Wien, Österreich. Online unter: http://www.freizeitforschung.at/data/forschungsarchiv.html (letzter Zugriff: 12.05.2020).

Open Access Dieses Buch wird unter der Creative Commons Namensnennung 4.0 International Lizenz (http://creativecommons.org/licenses/by/4.0/deed.de) veröffentlicht, welche die Nutzung, Vervielfältigung, Bearbeitung, Verbreitung und Wiedergabe in jeglichem Medium und Format erlaubt, sofern Sie den/die ursprünglichen Autor(en) und die Quelle ordnungsgemäß nennen, einen Link zur Creative Commons Lizenz beifügen und angeben, ob Änderungen vorgenommen wurden.

Die in diesem Buch enthaltenen Bilder und sonstiges Drittmaterial unterliegen ebenfalls der genannten Creative Commons Lizenz, sofern sich aus der Abbildungslegende nichts anderes ergibt. Sofern das betreffende Material nicht unter der genannten Creative Commons Lizenz steht und die betreffende Handlung nicht nach gesetzlichen Vorschriften erlaubt ist, ist für die oben aufgeführten Weiterverwendungen des Materials die Einwilligung des jeweiligen Rechteinhabers einzuholen.

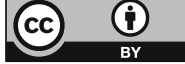

